\section{(2) OPEN ACCESS}

\title{
Lyso-Gb3 associates with adverse long-term outcome in patients with Fabry disease
}

\author{
Albina Nowak 다, 1,2 Felix Beuschlein, ${ }^{1}$ Visnuka Sivasubramaniam, ${ }^{1}$ David Kasper, ${ }^{3}$ \\ David G Warnock ${ }^{4}$
}

- Additional material is published online only. To view please visit the journal online (http://dx.doi.org/10.1136/ jmedgenet-2020-107338).

${ }^{1}$ Department of Endocrinology and Clinical Nutrition, University Hospital Zurich, Zurich, Switzerland

2Division of Internal Medicine Psychiatric University Hospital Zurich, Zurich, Switzerland ${ }^{3}$ ARCHIMED Life Science GmbH, Vienna, Austria

${ }^{4}$ Department of Medicine, Division of Nephrology, University of Alabama at Birmingham, Birmingham, Alabama, USA

\section{Correspondence to} Dr Albina Nowak, Department of Endocrinology and Clinical Nutrition, University Hospital Zurich and University of Zurich, 8091 Zürich, Switzerland; albina.nowak@usz.ch

Received 13 July 2020 Revised 6 November 2020 Accepted 3 December 2020

\begin{abstract}
Background Fabry disease (FD) is a rare X-linked lysosomal storage disease caused by mutations in the $\alpha$-galactosidase A gene (GLA) leading to deficiency of $\alpha$-galactosidase $A$ and ultimately in progressive glycosphingolipid accumulation, especially globotriaosylceramide (Gb3) and its deacylated derivative globotriaosylsphingosine (Lyso-Gb3). The aim of the study was to assess plasma Lyso-Gb3 levels as a possible factor associated with adverse outcomes in FD.
\end{abstract}

Methods In a cohort of 66 patients with genetically confirmed FD (26 males and 40 females), we analysed serum Lyso-Gb3 as a factor associated with adverse clinical outcomes in a long-term study. The main outcome was a composite endpoint of incident kidney replacement therapy, atrial fibrillation, pacemaker and/ or implantable cardioverter defibrillator, cerebrovascular events or death, whichever occurred first.

Results During the median follow-up time of 68 (40-80) months, events occurred in $19(29 \%)$ of the patients. In a Cox multivariate regression analysis, LysoGb3 levels (HR 4.62 (1.55 to 13.81); $p=0.006$ ) and the pretreatment exposure to Lyso-Gb3 (HR 3.41 (1.11 to 10.49); $p=0.03$ ) (both per SD increase) were significantly associated with adverse outcomes. If pretreatment Lyso-Gb3 exposure was added to multivariable logistic regression models containing age, sex, phenotype and enzyme replacement therapy as other covariates with the composite outcome as dependent variable, the area under the curve for the composite outcome significantly improved from 0.72 to 0.86 ( $\mathrm{p}$ comparison $=0.04$ ).

Conclusion Lyso-Gb3 is a significant risk factor associated with important clinical events. Whether treatment-related amelioration of Lyso-Gb3 levels will be associated with improved long-term outcome needs to be established in prospective intervention trials.

\section{INTRODUCTION}

Fabry disease (FD) is a rare X-linked glycosphingolipid storage disease and a result of mutations in the $\alpha$-galactosidase A gene (GLA), which leads to reduced activity of the encoded lysosomal exoglycohydrolase, $\alpha$-galactosidase A ( $\alpha$-Gal A) (EC 3.2.1.22; $\alpha$-Gal A). ${ }^{12}$ The $\alpha$-Gal A defect causes the progressive accumulation of globotriaosylceramide (Gb3) and other related glycosphingolipids in lysosomes of patients affected with FD. ${ }^{1}$

The clinical phenotypes include earlier-onset classic, and the attenuated, so-called later-onset variants. ${ }^{13-5}$ In the classic phenotype, males with little or no residual $\alpha$-Gal A activity develop marked intracellular Gb3-accumulation, with disease signs and symptoms, such as acroparesthesias, angiokeratoma, corneal opacities, hypohidrosis and gastrointestinal symptoms, occurring in childhood or early adolescence. With advancing age, the progressive deposition of glycosphingolipids, particularly in vascular endothelial lysosomes, cardiomyocytes and kidney podocytes is associated with left ventricular hypertrophy, hypertrophic cardiomyopathy, arrhythmias, kidney failure and cerebrovascular disease. This results in early demise, typically in the fourth or fifth decades of life. ${ }^{67}$ In contrast, males with the later-onset phenotype have residual $\alpha$-Gal A activity, no microvascular endothelial glycosphingolipid accumulation, and lack the early manifestations seen in classic male Fabry patients. They present in adulthood with cardiac or, less frequently, with kidney disease. ${ }^{38-10}$ Notably, a mild biopsy-proven Fabry nephropathy has also been reported in the cardiac subtype of the attenuated phenotype. ${ }^{11}$ Female heterozygotes have variable expression of $\mathrm{FD}$, ranging from asymptomatic to severe, primarily associated with skewed X-chromosomal inactivation. ${ }^{12}$

Recently, the deacylated derivative of Gb3, globotriaosylsphingosine (Lyso-Gb3), has been identified as a hallmark of FD. ${ }^{13}$ Nonetheless, it remained highly debated, whether Lyso-Gb3 is an independent risk factor for unfavourable clinical events. Arends and colleagues assessed the event-free survival time from birth until the first clinical event using plasma Lyso-Gb3 at the first clinic assessment. ${ }^{5}$ Thereby, the authors conducted a retrospective analysis rather than a prospective mortality study because measurements of LysoGb3 concentrations had only recently been developed and established. ${ }^{13}$ Based on these data for a combined cohort of patients with FD, higher Lyso-Gb3 concentrations at first clinical visit were found to be associated with clinical event rate occurring between birth and the time of the Lyso-Gb3 measurement. In contrast, analysing Lyso-Gb3 concentrations only for classic males and females, its levels were not significantly associated with previous clinical event rates, although classic patients more frequently develop clinical events in prospective survival analyses ${ }^{5}$ and suffer from a more severe phenotype. This lack of clarity reduces the usefulness of Lyso-Gb3 as a biomarker for severity and progression of FD.

Early and accurate risk stratification of patients with FD remains an unmet clinical need. Herein, 
we have assessed serum Lyso-Gb3 levels as a possible factor associated with adverse outcomes in a prospective long-term study of a large and well-defined population of genetically confirmed Fabry patients. Importantly, all Lyso-Gb3 measurements were performed by the same laboratory and using the same analytical standards, ${ }^{414}$ and their levels as well as clinical covariates were stratified by gender and phenotype.

\section{METHODS}

\section{Study population and treatment}

This is a retrospective analysis of a prospective cohort; samples for the Lyso-Gb3 concentrations were prospectively drawn during the routine annual examinations. The entire study period was between September 2013 and April 2020. The study was conducted in accordance with the principles of the Helsinki Declaration. Informed consent for collecting clinical data and blood samples was obtained from all patients. All authors have read and approved the manuscript.

All patients had a confirmed GLA mutation diagnosis and presented for routine annual examinations at our FD centre. The cohort was established in 2001 when enzyme replacement therapy (ERT) was approved and offered to patients with FD. ERT was prescribed at the licensed dose of either $0.2 \mathrm{mg} / \mathrm{kg}$ body weight of recombinant agalsidase- $\alpha$ (Replagal) or $1 \mathrm{mg} / \mathrm{kg}$ body weight agalsidase- $\beta$ (Fabrazyme) and given intravenously biweekly. ERT was initiated according to the written local guidelines and prescribed as reported previously. ${ }^{15}$

We measured Lyso-Gb3 in 66 adult patients (males: $n=26$ (39\%)) at the University Hospital Zurich, Switzerland, between September 2013 and December 2016. If patients had more than one Lyso-Gb3 measurement, the first result was used for the present longitudinal survival analysis. All clinical data were extracted from medical records, and the adverse events were evaluated during annual examinations as reported previously. ${ }^{15}$

\section{Phenotyping}

All mutations have been classified as coding for the classic or later-onset phenotype based on genotype and residual $\alpha-\mathrm{Gal} A$ activity in males and are published in the International Fabry Disease Genotype/Phenotype Database (www.dbFGP.org) and in previous studies. ${ }^{414}$ Nonsense, frameshift, consensus splice site (mutations that can affect the splice-donor and splice-acceptor sequences and alter the mature messenger RNA, leading to the production of abnormal proteins ${ }^{16}$ ) and some missense mutations encode for $0 \%$ to $2 \%$ residual $\alpha$-Gal A activity and lead to the classic phenotype in males. Alternative splicing mutations and certain other missense mutations encode for $>2 \%$ of mean normal $\alpha$-Gal A activity and cause attenuated, the so-called later-onset phenotype in males. The phenotypic assignments of the mutations are supported by the clinical manifestations in males, the age of symptom onset and by in vitro expression assays as reported previously. ${ }^{17} 18$

\section{Lyso-Gb3 measurement}

For serum Lyso-Gb3 levels, blood samples were centrifuged, and serum was immediately frozen at $-80^{\circ} \mathrm{C}$ for a later batch analysis. The samples were measured by high-sensitive electrospray ionisation liquid chromatography tandem mass spectrometry using an adapted method from Gold. A 7-point serum calibrator and an internal standard for Lyso-Gb3 quantification (covering the analytic range from 0 to $120 \mathrm{ng} / \mathrm{mL}$; lower limit of quantification: $0.3 \mathrm{ng} / \mathrm{mL}$ ) and three-level controls (3, 30 and $100 \mathrm{ng} /$ $\mathrm{mL}$ ) for quality control were used (ARCHIMED Life Science
GmbH, Vienna, Austria; www.archimedlife.com) as previously reported. $^{14}$

\section{Endpoint definition and evaluation}

As a primary endpoint, we defined the composite of requiring kidney replacement therapy (RRT) (kidney transplantation or chronic dialysis), newly diagnosed atrial fibrillation (AF) of any type (paroxysmal/persistent), pacemaker and/or implantable cardioverter defibrillator (ICD) implantation, cerebrovascular events (stroke or transient ischaemic attack (TIA)) diagnosed by a neurologist and death, whichever occurred first after the baseline serum Lyso-Gb3 measurement. These endpoints, with the exception of death, were prospectively defined according to Arends et al..$^{5}$ In contrast to this study, death was added to the primary endpoint because of the prospective observational period of our study, while this study retrospectively assessed the clinical event rates from birth to first visit. It is important to note that this previous study did not conduct a mortality study, because their patients were included at first visit and retrospectively analysed. ${ }^{5}$ In our study, the follow-up was censored at the date of the first event after the baseline Lyso-Gb3 measurement to calculate HRs. The follow-up time for patients without events was censored at the 1 April 2020. If the patients died outside of the Fabry Centre, the date of death was evaluated by asking the responsible general practitioner, the family or the nurse administering ERT in the home care setting.

Serum level Lyso-Gb3 at the first measurement (1) and the exposure to Lyso-Gb3 before disease-specific treatment (2) were employed as primary exposure variables. To calculate the cumulative pretreatment exposure to Lyso-Gb3, its serum levels measured at baseline of the observational period, all measured after September 2013, were multiplied by the age in years of the patients at ERT initiation, which was between 2001 and 2019. Additionally, the exposure to Lyso-Gb3 before diagnosis of FD, calculated as product of Lyso-Gb3 levels at baseline and age at diagnosis, was employed as exploratory variable. We did so under a simplified assumption that the levels of Lyso-Gb3 are linear as function of years of age.

\section{Definition of binary covariates}

The Lyso-Gb3 measures were classified by their median values, stratified by gender and Fabry phenotype (ie, classic vs lateronset) to generate a binary covariate with ' 0 ' corresponding to female gender, later-onset phenotype and Lyso-Gb3 level below the median value for each gender and phenotype strata. The other binary covariates included median age stratified by gender and phenotype at the baseline evaluation, gender, treatment with ERT at the time of baseline evaluation and Fabry phenotype.

\section{Statistical analysis}

We used descriptive statistics for the baseline characteristics at the time of the first Lyso-Gb3 measurement. Categorical variables were expressed as proportions, continuous variables as means with SD or medians with IQRs. Normal distribution was assessed by Shapiro-Wilk test. The primary exposure variables showed a non-parametric distribution and, therefore, were logtransformed for modelling. Comparison of Lyso-Gb3 groups according to sex and phenotype was made using one-way analysis of variance test.

Kaplan-Meier analysis was performed and log-rank test of survival distributions equality for patients with baseline serum level Lyso-Gb3 at the first measurement (1) and the exposure 
to Lyso-Gb3 before disease-specific treatment (2) above versus below median were calculated.

Cox regression analysis was used to examine the mortality risk associated with Lyso-Gb3 at the first measurement (1) and the exposure to Lyso-Gb3 before disease-specific treatment (2). Multivariable models were applied to adjust for potential confounders using prior knowledge of variables that have been associated with risk in patients with FD in previous studies. ${ }^{5} \mathrm{We}$ defined model 1 as a hierarchical adjustment for age; model 2 for male sex; model 3 for the classic phenotype. These models were used for all multivariable Cox regression analyses.

Receiver-operating characteristic (ROC) curves were generated with multifactor logistic regression models, and the effect of adding a specific covariate was evaluated by testing for significant change in the area under the ROC curve (AUC). The individual contributions of two different measures of Lyso-Gb3 concentrations (baseline Lyso-Gb3 and cumulative Lyso-Gb3 exposure) as well as a comparison of the contribution of each Lyso-Gb3 measure was assessed.

The ROC curves were generated and compared using Stata V.16.1 and the roccomp routine. The other statistical analyses were performed using the SPSS/PC (V.26.0; SPSS). All statistical tests were two-sided, and $\mathrm{p}$ values $<0.05$ were considered significant.

\section{RESULTS}

The clinical characteristics at the time of the first Lyso-Gb3 measurement in all patients according to sex and phenotype are shown in online supplemental table 1, the detailed baseline demographical and biochemical characteristics in online supplemental table 2 .

In total, 66 patients ( 26 males and 40 females) with the median age of 44 years were included in the analysis. The highest LysoGb3 levels were measured in the classic males, followed by the classic heterozygous females. All patients on RRT were male. Of 50 patients on ERT, 6 were treated with agalsidase- $\beta, 44$ with agalsidase- $\alpha$, all at the licensed full dose of each preparation.

\section{Events}

No patients were lost to follow-up. During the median follow-up time of 68 (40-80) months, the first event occurred in 19 (29\%) of the patient population. The clinical outcomes are summarised in table 1 and are shown in detail in online supplemental table 3 . Five classic patients (males $n=4$, females $n=1$ ) and none lateronset patients died during the observational period. Clinical events occurred in 18 classic patients (males $n=9$, females $n=9$ ) and in one later-onset male (table 1).

Seven patients underwent pacemaker and/or ICD implantation: three because of a high-degree atrioventricular block, four as primary prophylaxis due to high-degree myocardial hypertrophy and fibrosis; in three patients, a new-onset of AF occurred; one

\begin{tabular}{lllll}
\hline Table 1 & Clinical outcomes & & \\
& $\begin{array}{l}\text { Classic } \\
\text { males } \\
\mathrm{N}=21\end{array}$ & $\begin{array}{l}\text { Classic } \\
\text { females } \\
\mathrm{N}=36\end{array}$ & $\begin{array}{l}\text { Later-onset } \\
\text { males } \\
\mathrm{N}=5\end{array}$ & $\begin{array}{l}\text { Later-onset } \\
\text { females } \\
\mathrm{N}=4\end{array}$ \\
\hline $\begin{array}{l}\text { Death, } \mathrm{n}(\%) \\
\begin{array}{l}\text { Composite outcome* } \\
\mathrm{n}(\%)\end{array}\end{array}$ & $9(20)$ & $1(2.8)$ & $0(0)$ & $0(0)$ \\
\hline
\end{tabular}

${ }^{*}$ Composite of first occurrence of kidney replacement therapy requirement (kidney transplant or chronic dialysis), new onset of atrial fibrillation, pacemaker and/or implantable cardioverter defibrillator implantation, myocardial infarction, cerebrovascular events (stroke or transient ischaemic attack), death.
Table 2 HRs* (and 95\% Cls) for occurrence of primary endpoint† according to the baseline serum Lyso-Gb3 levels and the pretreatment Lyso-Gb3 exposure, both per natural log increase

\begin{tabular}{|c|c|c|c|c|c|c|}
\hline & \multicolumn{3}{|c|}{ Baseline Lyso-Gb3 levels } & \multicolumn{3}{|c|}{ Pretreatment exposure to Lyso-Gb3 } \\
\hline & HR & $95 \% \mathrm{Cl}$ & $P$ value & HR & $95 \% \mathrm{Cl}$ & $P$ value \\
\hline Crude & 2.12 & 1.32 to 3.42 & 0.002 & 3.02 & 1.69 to 5.37 & $<0.001$ \\
\hline Model 1 & 2.45 & 1.44 to 4.14 & 0.001 & 2.88 & 1.58 to 5.25 & 0.001 \\
\hline Model 2 & 3.75 & 1.44 to 9.73 & 0.007 & 3.69 & 1.60 to 8.51 & 0.002 \\
\hline Model 3 & 4.62 & 1.55 to 13.81 & 0.006 & 3.41 & 1.11 to 10.49 & 0.03 \\
\hline
\end{tabular}

patient received a kidney transplantation, one went on chronic dialysis; in one, a stroke occurred; one suffered myocardial infarction, five patients died. Overall, 21 events occurred in the whole population: one female with pacemaker implantation as first event subsequently suffered from two strokes. The distribution of the incident composite outcome and, separately, death during the observational period, according to sex and phenotype, is shown in table 2 . This table also summarises the late disease complications at baseline, when plasma Lyso-Gb3 levels were measured.

In males and females, serum levels of Lyso-Gb3 were significantly higher in patients who developed events versus patients without events (figure 1).

In the Kaplan-Meier analysis, the median of plasma Lyso-Gb3 levels and of the cumulative pretreatment Lyso-Gb3 exposure (figure 2), better than age, sex and phenotype (online supplemental figure 1), separated patients with versus without clinical events.

In the Cox regression analysis, Lyso-Gb3 levels and the pretreatment Lyso-Gb3 exposure were significantly associated with the adverse outcomes in the crude model and after the adjustment for age in model 1, male sex in model 2 and classic phenotype in model 3 . This association remained unchanged after the exclusion of the six patients with agalsidase- $\beta$ : for LysoGb3 levels HR 2.26 (1.38 to 3.71), $\mathrm{p}=0.001$ in crude model; HR 2.50 (1.48 to 4.27), $\mathrm{p}=0.001$ in model 1 ; HR 4.36 (1.61 to 11.80), $\mathrm{p}=0.004$ in model 2 ; HR 5.53 (1.80 to 17.00), $\mathrm{p}=0.003$ in model 3; for the pretreatment exposure to Lyso-Gb3 HR 2.93 (1.68 to 5.12), $\mathrm{p}<0.001$ in crude model; HR 2.76 (1.54 to 4.96), $\mathrm{p}=0.001$ in model 1 ; HR 3.82 (1.66 to 8.79), $\mathrm{p}=0.002$ in model 2; HR 3.59 (1.19 to 10.84), $\mathrm{p}=0.02$ in model 3 (all per SD increase). In addition, the cumulative exposure to LysoGb3 before Fabry diagnosis was significantly associated with the adverse outcomes: HR 2.07 (1.52 to 2.82), $\mathrm{p}<0.001$ in crude model; HR 2.01 (1.47 to 2.77), $\mathrm{p}<0.001$ in model 1; HR 2.21 (1.39 to 3.50$), p=0.001$ in model 2 ; HR 2.15 (1.31 to 3.53), $\mathrm{p}=0.003$ in model 3 (all per SD increase).

The ROC curve indicated a moderate to high association of baseline Lyso-Gb3 and the pretreatment Lyso-Gb3 exposure with the composite outcome (figure 3).

If pretreatment Lyso-Gb3 exposure was added to the multivariable logistic regression model containing age, sex, phenotype and ERT as other covariates and the composite outcome as dependent variable, the AUC for the composite outcome significantly improved from 0.72 to 0.86 ( $\mathrm{p}$ comparison $=0.04$ ).

If baseline Lyso-Gb3 levels were added to the multivariable logistic regression model containing the same covariates as above, the AUC for the composite outcome improved not significantly from 0.72 to 0.77 ( $\mathrm{p}$ comparison $=0.16$ ). 


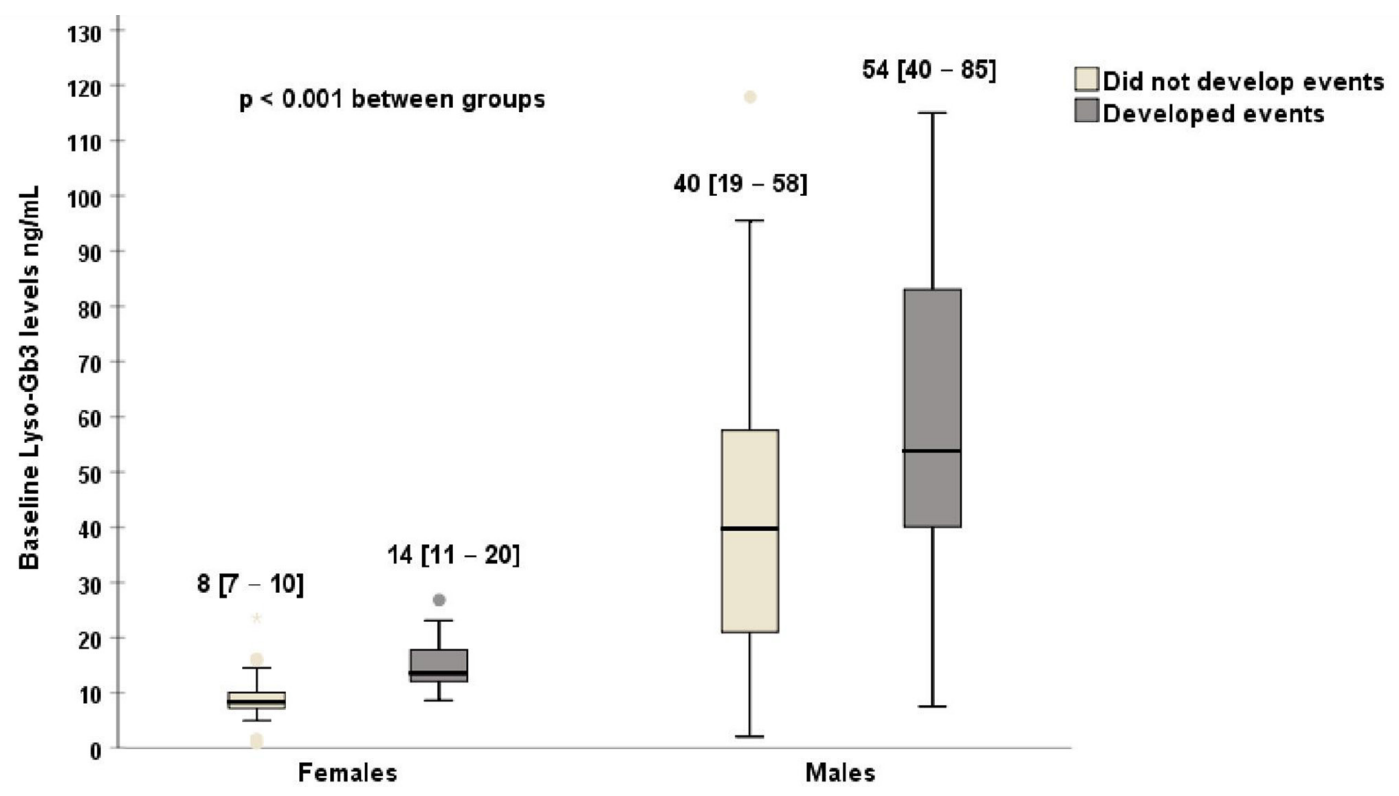

Figure 1 Baseline serum Lyso-Gb3 levels in patients who developed events versus no events during the observational period. Comparison of Lyso-Gb3 groups according to sex and phenotype was made using one-way analysis of variance test. The horizontal lines within the boxplots represent medians, the open circles outliers; the star represents an extreme outlier. The whiskers confine the 1.5 distance of the IQRs, respectively.

\section{DISCUSSION}

In this relatively large well-characterised cohort of genetically proven patients with FD, higher baseline levels of Lyso-Gb3 were associated with a higher risk of complications such as death, stroke/TIA, new manifestation of AF, requirement for RRT, pacemaker or ICD implantation. More importantly, the cumulative exposure to Lyso-Gb3 during the untreated period seems to predispose patients with FD to worse clinical outcomes.

These findings correspond to the fact that Lyso-Gb3 reflects severity of FD, shown by us ${ }^{414}$ and other groups, ${ }^{19}{ }^{20}$ and to the fact that Lyso-Gb3 levels in serum correspond to the Gb3 burden in the vital organs. ${ }^{13}$ In FD, the correct risk stratification based on an understanding of the genotype and phenotype relationship is an urgent but still unmet clinical need. An increased awareness of FD among primary care physicians and specialists as well as systematic screening among high-risk populations ${ }^{21}$ and newborns ${ }^{22}$ has been becoming increasingly frequent, leading to an increased detection of mutations with unknown clinical progression. ${ }^{23}$ A specific biomarker could not only fill the diagnostic gap but also improve disease staging and risk stratification as well as support the decision whether a patient should be started on ERT and or if he should be more closely monitored. Of note, patients with FD with low or undetectable Lyso-Gb3 levels can still suffer adverse events because of possibly having genetic and epigenetic modifiers of their phenotype, additional diseases, environmental conditions such as toxins or lifestyle modalities. Thus, no biomarker can replace a thorough clinical multidisciplinary characterisation, genetic testing and the phenotypic descriptions of the family members.

Lyso-Gb3 has been described as a helpful tool for newborn ${ }^{22}$ and high-risk group screening, ${ }^{21} 25$ for diagnosis, ${ }^{26-29}$ as a biomarker for phenotypic assignment, ${ }^{14}$ a tool reflecting disease severity ${ }^{4}$ and therapeutic monitoring. ${ }^{30-32}$ As a result of enzymatic deficiency, Gb3 accumulation occurs in classic phenotype males, already before symptom manifestation. ${ }^{33} 34$ Lyso-Gb3 may originate from deacylation of the stored Gb3 or be synthesised by sequential glycosylation of accumulating sphingolipid precursors. ${ }^{13}$ As suggested by our results, the toxic effects of
Lyso-Gb3 seem to cumulate during the pretreatment period. A possible explanation could be that Lyso-Gb3 might not only act as a marker of disease severity but also have direct toxic effects as a metabolite. Supporting this, Lyso-Gb3 has been demonstrated to promote Notch1-mediated inflammatory and fibrogenic response in podocytes, potentially contributing to Fabry nephropathy. ${ }^{35}$ In studies by Sanchez-Niño and colleagues, Lyso-Gb3 has been further shown to disrupt the gut microbiota homeostasis modulating the biofilm formation of healthy enteric bacteria, potentially enhancing the gastrointestinal symptoms of patients with FD. ${ }^{36} 37$ Furthermore, plantar administration of Lyso-Gb3 stimulated allodynia in healthy mice; in vitro application of Lyso-Gb3 activated $\mathrm{Ca}^{2+}$ uptake of voltage-dependent nociceptive neurons, suggesting that this toxic metabolite may induce pain through direct actions on sensory afferents. ${ }^{38}$ Moreover, Aerts and colleagues observed that concentrations of Lyso-Gb3, similar to those observed in plasma of symptomatic Fabry patients promoted proliferation of smooth muscle cells in culture. $^{13}$

Arends and colleagues observed that ERT initiation at a younger age resulted in a more pronounced sustainable decrease of Lyso-Gb3, in classic males. ${ }^{31}$ Noteworthy, plasma Lyso-Gb3 adequately reflects the cumulative lifetime treatment effect. If adding cumulative pretreatment Lyso-Gb3 exposure to the logistic regression model containing other important risk factors such as age, sex, phenotype and ERT requirement, the AUC for the composite outcome significantly improved. Our studies are the first to analyse the cumulative pretreatment toxic potential of this metabolite. The consequence of this knowledge could be that disease-specific treatment aiming at decrease of Lyso-Gb3 could lower the incidence of the composite endpoint, which could be shown by future studies. A possible design of a LysoGb3-guided study could be to administer two different doses of ERT, which, in the absence of antibodies, would lead to a greater decrease of Lyso-Gb3 ${ }^{30} 32$ levels and, presumably, to a lower incidence of clinical events, in patients with the higher dose.

The strength of these studies includes a long observational period for relevant clinical outcomes in a genetically confirmed one-country 

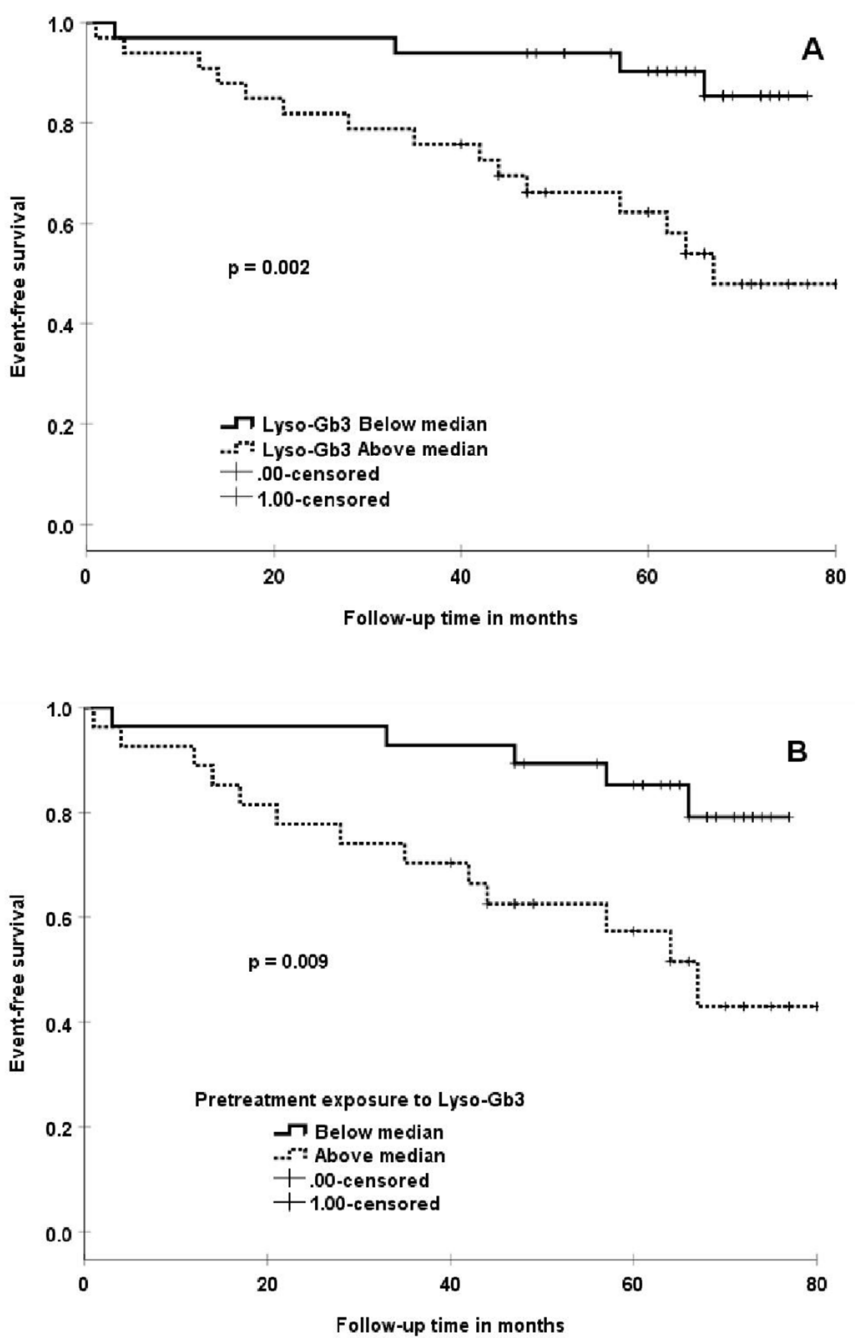

Figure 2 Time to first complication in patients with Fabry disease according to serum Lyso-Gb3 levels (A) and the pretreatment exposure to Lyso-Gb3 (B), grouped as above andbelow median. The diagram shows the results of Kaplan-Meier analysis and log-rank test of survival distribution equality.

cohort, where patients are treated similarly and exposed to similar environmental conditions and complete follow-up data. The latter is due to Swiss regulation that the ERT prescription and patient's follow-up remain reserved for the Fabry Centre. Furthermore, an under-reporting of clinical events is unlikely in a setting like this, where a cohort is personally followed and treated at the same specialised centre and by the same treating physician. Disadvantages like under-reporting of clinical events and lost to follow-up can sometimes limit large registry-based studies. ${ }^{39}$ In addition, the LysoGb3 measurements were conducted at one laboratory with the same standards of the technical methods.

These studies are also limited since we analysed the cumulative longterm effects of Lyso-Gb3 under the assumption that its levels increase with age in a horizontal linear manner. However, Lyso-Gb3, already increased in classic Fabry male newborns, ${ }^{13}{ }^{40}$ continues rising during the presymptomatic phase, representing a metabolic progression to the clinical phenotype, and reaches its plateau at the advent of the early symptoms. ${ }^{33}$ Then, it rapidly decreases by approximately $70 \%$ after 2-3 months of ERT initiation, remaining stable but elevated, in patients without antibodies against ERT. ${ }^{30}$ In contrast, in patients with antibodies, Lyso-Gb3 gradually increases again after a decrease, following ERT initiation. ${ }^{32}$ In migalastat-treated patients, Lyso-Gb3

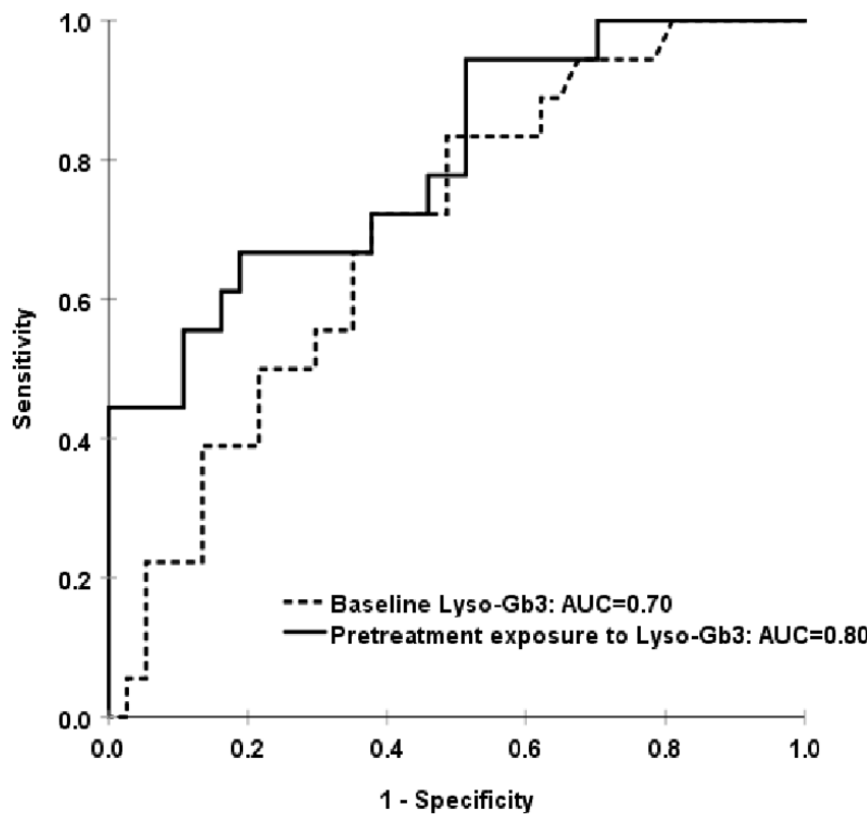

Figure 3 Accuracy of baseline Lyso-Gb3 levels and the cumulative pretreatment exposure to Lyso-Gb3 for the association with the adverse outcomes. The diagram represents receiver-operating characteristic curves. $A U C$, area under the curve.

increases, decreases or remains stable dependent of the degree of mutation amenability. ${ }^{41}$ In our studies, we had to simplify the course of Lyso-Gb3 as linear for the calculation of cumulative Lyso-Gb3 exposure because Lyso-Gb3 had been discovered as a hallmark of FD in $2007^{13}$ and reliable laboratory measurements were available even later and were applied in this cohort of adult patients starting from 2013. ${ }^{14}$ Although, Lyso-Gb3 decreases after ERT initiation, ${ }^{30} \mathrm{a}$ simplified assumption of linearity seems to be useful to estimate the pretreatment cumulative effects to Lyso-Gb3 because (1) ERT was initiated relatively late-in the adult age-in this old Fabry cohort, and because (2), following ERT, Lyso-Gb3 decreases but remains greatly elevated above the normal range. ${ }^{30}$ Of note, we classified the phenotype as published in the International Fabry Disease Genotype/ Phenotype Database (www.dbFGP.org) for all mutations reported in this study. However, it needs to be mentioned that patients with the same genotype, sex and similar age can have phenotypic variations as previously shown in our ${ }^{4}$ and other cohorts, ${ }^{42}$ possibly caused by concomitant mutations, epigenetic phenomena and environmental conditions. Further limitation is that our results are not entirely in line with previous reports that found Lyso-Gb3 not to be associated with clinical events. Arends and colleagues analysed Lyso-Gb3 concentrations only for classic males and females, its levels were not significantly associated with previous clinical event rates. ${ }^{5}$ Further limitation is that the number of patients is relatively low due to the nature of a rare disease. Due to this, a predefined composite outcome rather than mortality alone was used to assess risk factors. Therefore, further studies are needed to confirm our findings. Finally, for the use as a clinical biomarker, standardisation of the technical methods and interlaboratory testing is needed in order to compare Lyso-Gb3 measurements among laboratories.

In conclusion, the major metabolite of FD Lyso-Gb3 appears as a potential marker of disease progression and is associated with increased risk of mortality and other significant clinical events. The cumulative pretreatment exposure to Lyso-Gb3 seems to worsen the long-term clinical outcomes, while the disease-specific treatment should ameliorate cumulative toxic Lyso-Gb3 effects. 
Correction notice This article has been corrected since it first published. The provenance and peer review statement has been included.

Contributors AN contributed to conception and design of the work, acquisition, analysis and interpretation of data and drafting the manuscript. FB contributed to interpretation of data and final approval of the last version. VS and DK contributed to acquisition of data, interpretation of data and final approval of the last version. DGW contributed to design of the work, acquisition, analysis and interpretation of data and final approval of the last version.

Funding The Lyso-Gb3 measurements were determined by ARCHIMED Life Science, Vienna, Austria. The ARCHIMED Life Science laboratory member DCK participated in writing and approving the manuscript. The laboratory members were blinded to patients' names and all clinical and biochemical information and had no role in the collection of samples, interpretation of data and the decision to submit the article for publication.

Competing interests $A N$ received lecturing honoraria and research support from Sanofi Genzyme, Takeda and Amicus and received financial publication support for this article from Sanofi Genzyme. DGW serves as a consultant for Amicus, Protalix, and Chiesi Pharma.

\section{Patient consent for publication Not required.}

Ethics approval The study was approved by the Zurich Ethics Committee.

Provenance and peer review Not commissioned; externally peer reviewed.

Data availability statement Data are available upon reasonable request. All data relevant to the study are included in the article or uploaded as supplementary information. Deidentified participant data is available from the first author upon reasonable request as a data file under the email address albina.nowak@usz.ch.

Supplemental material This content has been supplied by the author(s). It has not been vetted by BMJ Publishing Group Limited (BMJ) and may not have been peer-reviewed. Any opinions or recommendations discussed are solely those of the author(s) and are not endorsed by BMJ. BMJ disclaims all liability and responsibility arising from any reliance placed on the content. Where the content includes any translated material, BMJ does not warrant the accuracy and reliability of the translations (including but not limited to local regulations, clinical guidelines, terminology, drug names and drug dosages), and is not responsible for any error and/or omissions arising from translation and adaptation or otherwise.

Open access This is an open access article distributed in accordance with the Creative Commons Attribution Non Commercial (CC BY-NC 4.0) license, which permits others to distribute, remix, adapt, build upon this work non-commercially, and license their derivative works on different terms, provided the original work is properly cited, appropriate credit is given, any changes made indicated, and the use is non-commercial. See: http://creativecommons.org/licenses/by-nc/4.0/

\section{ORCID iD}

Albina Nowak http://orcid.org/0000-0002-4119-5516

\section{REFERENCES}

1 Desnick R, loannou, C Y. Eng, $\alpha$-galactosidase A deficiency: Fabry disease. In: Scriver CR, Beaudet AL, Sly WS, Valle D, eds. The metabolic and molecular bases of inherited disease. New York: McGraw-Hill, 2001: 3733-74.

2 Desnick RJ, Brady R, Barranger J, Collins AJ, Germain DP, Goldman M, Grabowski G, Packman S, Wilcox WR. Fabry disease, an under-recognized multisystemic disorder: expert recommendations for diagnosis, management, and enzyme replacement therapy. Ann Intern Med 2003;138:338-46.

3 von Scheidt W, Eng CM, Fitzmaurice TF, Erdmann E, Hübner G, Olsen EG, Christomanou H, Kandolf R, Bishop DF, Desnick RJ. An atypical variant of Fabry's disease with manifestations confined to the myocardium. N Engl J Med 1991;324:395-9.

4 Nowak A, Mechtler TP, Hornemann T, Gawinecka J, Theswet E, Hilz MJ, Kasper DC. Genotype, phenotype and disease severity reflected by serum LysoGb3 levels in patients with Fabry disease. Mol Genet Metab 2018;123:148-53.

5 Arends M, Wanner C, Hughes D, Mehta A, Oder D, Watkinson OT, Elliott PM, Linthorst GE, Wijburg FA, Biegstraaten M, Hollak CE. Characterization of classical and nonclassical Fabry disease: a multicenter study. J Am Soc Nephrol 2017;28:1631-41.

6 Schiffmann R, Warnock DG, Banikazemi M, Bultas J, Linthorst GE, Packman S, Sorensen SA, Wilcox WR, Desnick RJ. Fabry disease: progression of nephropathy, and prevalence of cardiac and cerebrovascular events before enzyme replacement therapy. Nephrol Dial Transplant 2009;24:2102-11.

$7 \mathrm{MacDermot}$ KD, Holmes A, Miners AH. Anderson-Fabry disease: clinical manifestations and impact of disease in a cohort of 60 obligate carrier females. J Med Genet 2001;38:769-75.
8 Nakao S, Takenaka T, Maeda M, Kodama C, Tanaka A, Tahara M, Yoshida A, Kuriyama M, Hayashibe H, Sakuraba H. An atypical variant of Fabry's disease in men with left ventricular hypertrophy. N Eng/ J Med 1995;333:288-93.

9 Nakao S, Kodama C, Takenaka T, Tanaka A, Yasumoto Y, Yoshida A, Kanzaki T, Enriquez ALD, Eng CM, Tanaka H, Tei C, Desnick RJ. Fabry disease: detection of undiagnosed hemodialysis patients and identification of a "renal variant" phenotype. Kidney Int 2003;64:801-7.

10 Shabbeer J, Yasuda M, Benson SD, Desnick RJ. Fabry disease: identification of 50 novel alpha-galactosidase A mutations causing the classic phenotype and three-dimensional structural analysis of 29 missense mutations. Hum Genomics 2006;2:297-309.

11 Oliveira JP, Nowak A, Barbey F, Torres M, Nunes JP, Teixeira-e-Costa F, Carvalho F, Sampaio S, Tavares I, Pereira O, Soares AL, Carmona C, Cardoso M-T, Jurca-Simina IE, Spada M, Ferreira S, Germain DP. Fabry disease caused by the Gla p.Phe113Leu (p.F113L) variant: natural history in males. Eur J Med Genet 2020;63:1037.

12 Echevarria L, Benistan K, Toussaint A, Dubourg O, Hagege AA, Eladari D, Jabbour F, Beldjord C, De Mazancourt P, Germain DP. X-Chromosome inactivation in female patients with Fabry disease. Clin Genet 2016;89:44-54.

13 Aerts JM, Groener JE, Kuiper S, Donker-Koopman WE, Strijland A, Ottenhoff R, van Roomen C, Mirzaian M, Wijburg FA, Linthorst GE, Vedder AC, Rombach SM, Cox-Brinkman J, Somerharju P, Boot RG, Hollak CE, Brady RO, Poorthuis BJ. Elevated globotriaosylsphingosine is a hallmark of Fabry disease. Proc Natl Acad Sci U S A 2008;105:2812-7.

14 Nowak A, Mechtler T, Kasper DC, Desnick RJ. Correlation of Lyso-Gb3 levels in dried blood spots and sera from patients with classic and Later-Onset Fabry disease. Mol Genet Metab 2017;121:320-4.

15 Siegenthaler M, Huynh-Do U, Krayenbuehl P, Pollock E, Widmer U, Debaix H, Olinger E, Frank M, Namdar M, Ruschitzka F, Nowak A. Impact of cardio-renal syndrome on adverse outcomes in patients with Fabry disease in a long-term follow-up. Int J Cardiol 2017;249:261-7.

16 Berget SM. Exon recognition in vertebrate splicing. J Biol Chem 1995;270:2411-4.

17 Benjamin ER, Della Valle MC, Wu X, Katz E, Pruthi F, Bond S, Bronfin B, Williams H, Yu J, Bichet DG, Germain DP, Giugliani R, Hughes D, Schiffmann R, Wilcox WR, Desnick RJ, Kirk J, Barth J, Barlow C, Valenzano KJ, Castelli J, Lockhart DJ. The validation of pharmacogenetics for the identification of Fabry patients to be treated with migalastat. Genet Med 2017:19:430-8.

18 Yasuda M, Shabbeer J, Osawa M, Desnick RJ. Fabry disease: novel alpha-galactosidase a 3'-terminal mutations result in multiple transcripts due to aberrant 3'-end formation. Am J Hum Genet 2003;73:162-73.

19 Arends M, Körver S, Hughes DA, Mehta A, Hollak CEM, Biegstraaten M. Phenotype, disease severity and pain are major determinants of quality of life in Fabry disease: results from a large multicenter cohort study. J Inherit Metab Dis 2018;41:141-9.

20 Rombach SM, Dekker N, Bouwman MG, Linthorst GE, Zwinderman AH, Wijburg FA, Kuiper S, Vd Bergh Weerman MA, Groener JEM, Poorthuis BJ, Hollak CEM, Aerts JMFG. Plasma globotriaosylsphingosine: diagnostic value and relation to clinical manifestations of Fabry disease. Biochim Biophys Acta 2010;1802:741-8.

21 Doheny D, Srinivasan R, Pagant S, Chen B, Yasuda M, Desnick RJ. Fabry Disease: prevalence of affected males and heterozygotes with pathogenic GLA mutations identified by screening renal, cardiac and stroke clinics, 1995-2017. J Med Genet 2018;55:261-8.

22 Wasserstein MP, Caggana M, Bailey SM, Desnick RJ, Edelmann L, Estrella L, Holzman I, Kelly NR, Kornreich R, Kupchik SG, Martin M, Nafday SM, Wasserman $R$, Yang $A$, Yu C, Orsini JJ. The new York pilot newborn screening program for lysosomal storage diseases: report of the first 65,000 infants. Genet Med 2019:21:631-40.

23 Smid BE, Hollak CEM, Poorthuis BJHM, van den Bergh Weerman MA, Florquin S, Kok WEM, Lekanne Deprez RH, Timmermans J, Linthorst GE. Diagnostic dilemmas in Fabry disease: a case series study on Gla mutations of unknown clinical significance. Clin Genet 2015;88:161-6.

24 Duro G, Zizzo C, Cammarata G, Burlina A, Burlina A, Polo G, Scalia S, Oliveri R, Sciarrino S, Francofonte D, Alessandro R, Pisani A, Palladino G, Napoletano R, Tenuta M, Masarone D, Limongelli G, Riccio E, Frustaci A, Chimenti C, Ferri C, Pieruzzi F, Pieroni M, Spada M, Castana C, Caserta M, Monte I, Rodolico MS, Feriozzi S, Battaglia Y, Amico L, Losi MA, Autore C, Lombardi M, Zoccali C, Testa A, Postorino M, Mignani R, Zachara E, Giordano A, Colomba P. Mutations in the Gla gene and LysoGb3: is it really Anderson-Fabry disease? Int J Mol Sci 2018;19:3726-3. doi:10.3390/ ijms 19123726

25 Auray-Blais C, Lavoie P, Abaoui M, Côté A-M, Boutin M, Akbari A, Levin A, Mac-Way $\mathrm{F}$, Tr Clarke J. High-Risk screening for Fabry disease in a Canadian cohort of chronic kidney disease patients. Clin Chim Acta 2020;501:234-40.

26 Niemann M, Rolfs A, Störk S, Bijnens B, Breunig F, Beer M, Ertl G, Wanner C, Weidemann F. Gene mutations versus clinically relevant phenotypes: lyso-Gb3 defines Fabry disease. Circ Cardiovasc Genet 2014;7:8-16.

27 Nowak A, Mechtler TP, Desnick RJ, Kasper DC. Plasma LysoGb3: a useful biomarker for the diagnosis and treatment of Fabry disease heterozygotes. Mol Genet Metab 2017:120:57-61.

28 Alharbi FJ, Baig S, Auray-Blais C, Boutin M, Ward DG, Wheeldon N, Steed R, Dawson C, Hughes D, Geberhiwot T. Globotriaosylsphingosine (Lyso-Gb) as a biomarker for cardiac variant (N215S) Fabry disease. J Inherit Metab Dis 2018;41:239-47. 
29 Baydakova GV, llyushkina AA, Moiseev S, Bychkov IO, Nikitina NV, Buruleva T A, Zakharova EY, N.V. Nikitina, C.A.C, Te Bcapital. $\alpha$-Galactosidase A/lysoGb3 ratio as a potential marker for Fabry disease in females. Clin Chim Acta 2020;501:27-32.

30 van Breemen MJ, Rombach SM, Dekker N, Poorthuis BJ, Linthorst GE, Zwinderman AH, Breunig F, Wanner C, Aerts JM, Hollak CE. Reduction of elevated plasma globotriaosylsphingosine in patients with classic Fabry disease following enzyme replacement therapy. Biochim Biophys Acta 2011;1812:70-6.

31 Arends M, Wijburg FA, Wanner C, Vaz FM, van Kuilenburg ABP, Hughes DA, Biegstraaten M, Mehta A, Hollak CEM, Langeveld M. Favourable effect of early versus late start of enzyme replacement therapy on plasma globotriaosylsphingosine levels in men with classical Fabry disease. Mol Genet Metab 2017;121:157-61.

32 Sakuraba H, Togawa T, Tsukimura T, Kato H. Plasma lyso-Gb3: a biomarker for monitoring Fabry patients during enzyme replacement therapy. Clin Exp Nephrol 2018;22:843-9.

33 Spada M, Kasper D, Pagliardini V, Biamino E, Giachero S, Porta F. Metabolic progression to clinical phenotype in classic Fabry disease. Ital I Pediatr 2017;43:1.

34 Vedder AC, Strijland A, vd Bergh Weerman MA, Florquin S, Aerts JMFG, Hollak CEM. Manifestations of Fabry disease in placental tissue. J Inherit Metab Dis 2006;29:106-11.

35 Sanchez-Niño MD, Carpio D, Sanz AB, Ruiz-Ortega M, Mezzano S, Ortiz A. Lyso-Gb3 activates Notch1 in human podocytes. Hum Mol Genet 2015;24:5720-32.
36 Sanchez-Niño MD, Aguilera-Correa J-J, Politei J, Esteban J, Requena T, Ortiz A. Unraveling the drivers and consequences of gut microbiota disruption in Fabry disease: the lyso-Gb3 link. Future Microbiol 2020;15:227-31.

37 Aquilera-Correa J-J, Madrazo-Clemente P, Martínez-Cuesta MDC, Peláez C, Ortiz A, Sánchez-Niño MD, Esteban J, Requena T. Lyso-Gb3 modulates the gut microbiota and decreases butyrate production. Sci Rep 2019;9:12010.

38 Choi L, Vernon J, Kopach O, Minett MS, Mills K, Clayton PT, Meert T, Wood JN. The Fabry disease-associated lipid Lyso-Gb3 enhances voltage-gated calcium currents in sensory neurons and causes pain. Neurosci Lett 2015;594:163-8.

39 Germain DP, Charrow J, Desnick RJ, Guffon N, Kempf J, Lachmann RH, Lemay R, Linthorst GE, Packman S, Scott CR, Waldek S, Warnock DG, Weinreb NJ, Wilcox WR. Ten-Year outcome of enzyme replacement therapy with agalsidase beta in patients with Fabry disease. J Med Genet 2015;52:353-8.

40 Chien Y-H, Bodamer OA, Chiang S-C, Mascher H, Hung C, Hwu W-L. Lysoglobotriaosylsphingosine (lyso-Gb3) levels in neonates and adults with the Fabry disease later-onset GLA IVS4+919G >A mutation. I Inherit Metab Dis 2013;36:881-5.

41 Nowak A, Huynh-Do U, Krayenbuehl Pierre-Alexandre, Beuschlein F, Schiffmann R, Barbey F. Fabry disease genotype, phenotype, and migalastat amenability: insights from a national cohort. J Inherit Metab Dis 2020:43:326-33.

42 Arends M, Biegstraaten M, Wanner C, Sirrs S, Mehta A, Elliott PM, Oder D, Watkinson OT, Bichet DG, Khan A, Iwanochko M, Vaz FM, van Kuilenburg ABP, West ML, Hughes DA, Hollak CEM. Agalsidase alfa versus agalsidase beta for the treatment of Fabry disease: an international cohort study. J Med Genet 2018;55:351-8. 